\title{
Avaliação dos processos de mistura de concretos permeáveis com seixo
}

\author{
Evaluation of mixing processes of pervious concrete with pebbles \\ Evaluación de procesos de mezcla de hormigón permeable con cantos rodados
}

Recebido: 23/03/2021 | Revisado: 29/03/2021 | Aceito: 02/04/2021 | Publicado: 12/04/2021

\author{
Juliana Chaves Cordeiro \\ ORCID: https://orcid.org/0000-0001-6811-4408 \\ Universidade Federal do Pará, Brasil \\ E-mail: jchaveseng2@gmail.com \\ Denny Martins Freitas \\ ORCID: https://orcid.org/0000-0002-9945-4410 \\ Universidade Federal do Pará, Brasil \\ E-mail: dennyn32@gmail.com \\ Luciana de Nazaré Pinheiro Cordeiro \\ ORCID: https://orcid.org/0000-0001-7931-4042 \\ Universidade Federal do Pará, Brasil \\ E-mail: lupcordeiroo@gmail.com \\ Arthur Aviz Palma e Silva \\ ORCID: https://orcid.org/0000-0001-5686-5984 \\ Universidade de Brasília, Brasil \\ E-mail: eng.aviz@gmail.com
}

\begin{abstract}
Resumo
A crescente impermeabilização dos espaços urbanos traz consigo problemas e desastres ambientais como as inundações, em decorrência da alteração na dinâmica da água da chuva que infiltrava e passa a escoar, exigindo a maior capacidade dos sistemas de micro e macrodrenagem. O concreto permeável (CPER), composto por cimento Portland e agregado graúdo, ganha espaço como forma de mitigar os efeitos da redução das áreas permeáveis. Nesse contexto, o presente trabalho busca investigar a influência da sequência de mistura no comportamento hidráulico e mecânico do CPER produzido com seixo rolado, dada a existência de lacunas na literatura sobre o impacto desse parâmetro sobre material. Para tal, foram estudadas três sequências de misturas a partir da produção de placas de CPER de dimensões $40 \mathrm{~cm} \times 40 \mathrm{~cm} \times 10 \mathrm{~cm}$. Foram analisados os resultados de resistência à tração na flexão, porosidade, tortuosidade e permeabilidade. Estatisticamente, não foram verificadas diferenças entre os resultados, entretanto, observou-se o crescimento do volume e da área de poros, alterando-se as sequencias, quando utilizado na produção dos concretos um misturador de eixo inclinado.
\end{abstract}

Palavras-chave: Concreto permeável; Sequência de mistura; Variável de controle.

\begin{abstract}
The increasing waterproofing of urban spaces brings environmental problems and disasters, such as flooding, resulting from changes in rainwater that seep and pass, requiring greater capacity of micro and macro drainage systems. Permeable concrete (CPER), composed of Portland cement and coarse aggregate, gains space as a way to mitigate the effects of reducing permeable areas. In this context, the present work seeks to investigate the influence of the mixing sequence on the hydraulic and mechanical behavior of the roll-milled CPER, given the presence of gaps in the literature about the impact of these parameters on the material. For this, three sequences of mixtures were studied from the production of CPER plates of dimensions $40 \mathrm{~cm} \times 40 \mathrm{~cm} \times 10 \mathrm{~cm}$. The results of resistance to flexural alteration, porosity, tortuosity and permeability were analyzed. Statistically, no differences were found between the results, however, reduced the volume growth and pore area, changing as sequences when used in the production of concrete or inclined shaft mixer.
\end{abstract}

Keywords: Permeable concrete; Mixing sequence; Control variable.

\section{Resumen}

La creciente impermeabilidad de los espacios urbanos trae consigo problemas ambientales y desastres como inundaciones, debido al cambio en la dinámica del agua de lluvia que se infiltra y comienza a drenar, requiriendo la mayor capacidad de los sistemas de micro y macrodrenaje. El hormigón permeable (CPER), compuesto por cemento Portland y árido grueso, gana espacio como forma de mitigar los efectos de la reducción de áreas permeables. En este contexto, el presente trabajo busca investigar la influencia de la secuencia de mezclado en el comportamiento hidráulico y mecánico del CPER producido con canto rodado, dada la existencia de vacíos en la literatura sobre el impacto de este parámetro en el material. Para ello, se estudiaron tres secuencias de mezclas a partir de la producción de placas CPER de dimensiones $40 \mathrm{~cm} \times 40 \mathrm{~cm} \times 10 \mathrm{~cm}$. Se analizaron los resultados de la resistencia a la tracción en flexión, porosidad, tortuosidad y permeabilidad. Estadísticamente, no hubo diferencias entre los resultados, sin 
embargo, sí hubo un aumento en el volumen y área de poro, cambiando las secuencias, al utilizar un mezclador de eje inclinado en la producción de hormigones.

Palabras clave: Hormigón permeable; Secuencia de mezcla; Control variable.

\section{Introdução}

O concreto permeável (CPER) vem sendo utilizado desde o século XX em países como Japão, EUA e Canadá, tendo como principais aplicações a fabricação de pré-moldados e a pavimentação de estacionamentos e calçadas. Nas últimas décadas, este material tem atraído interesse devido ao seu potencial como tecnologia resiliente, que visa mitigar os efeitos da urbanização sobre o meio ambiente, (Bhutta et al., 2015; ACI 522-R, 2010). O Pavimento convencional, muito utilizando em centros urbanos, é constituído de um revestimento com agregados (brita) e ligantes asfáltico, esse tipo de pavimento torna as superfícies trafegáveis por veículos altamente impermeáveis, atenuando o problema de alagamentos (Salomão et al. 2018)

O termo Concreto Permeável é utilizado para descrever um material composto por cimento, água e agregados graúdos com granulometria uniforme. A ausência ou redução da quantidade de finos e o baixo teor de pasta empregados em sua produção possibilitam a incorporação de um elevado volume de vazios na estrutura, geralmente em torno de $15 \%$ a $35 \%$ do volume total, com tamanhos variando entre $2 \mathrm{~mm}$ e $8 \mathrm{~mm}$ (Bonicelli et al., 2015; Zhong et al., 2016). Tal estrutura resulta em um material poroso com elevada condutibilidade hidráulica que deve atingir valores mínimos de permeabilidade ( $1 \mathrm{~mm} / \mathrm{s})$ e resistência (2 MPa, para tráfego leve) para que possa apresentar bom desempenho (ABNT/NBR 16416, 2015). Dada essa característica e sua aplicabilidade, o CPER tem a capacidade de reduzir o volume de água escoado superficialmente por infiltração, sendo listado pela Agência de Proteção Ambiental dos Estados Unidos (U.S. Environmental Protection Agency EPA) como uma das melhores práticas do gerenciamento do sistema de águas pluviais (Batezini, 2013).

De acordo com Chindaprasirt et. al (2008), os principais parâmetros que influenciam na obtenção de concretos permeáveis resistentes, duráveis e com adequado índice de vazios são o método de dosagem e os processos de mistura e compactação. Nesse contexto, diversos estudos têm sido realizados buscando verificar a influência de variáveis como a relação água cimento (Chindaprasirt et al.,2008), as características dos agregados (Cósic et al., 2015; Yahia et al.,2014), o teor e as propriedades da pasta na mistura (Torres et al., 2015), entretanto, a condição ótima para a produção do material não foi estabelecida e em muitos países, especialmente nos emergentes, sua implementação é limitada devido à falta de padronização de técnicas de preparo e de práticas construtivas (Chandrappa et al., 2016).

Diante do exposto, verifica-se que o estudo sobre CPER apresenta lacunas na literatura no que tange aos processos de mistura, variável que pode influenciar na trabalhabilidade, nas propriedades do material no estado fresco e endurecido e em sua durabilidade (ASTM C94, 2009). Buscando preencher tais lacunas, o presente trabalho tem por objetivo avaliar a influência dos processos de mistura no comportamento hidráulico e mecânico do material, para aplicação em tráfego leve. Para tal, analisou-se o comportamento de concretos formulados por diferentes ordens de mistura, com um traço determinado a partir do método indicado por Castro et. al (2009).

\section{Metodologia}

Este trabalho é de caráter laboratorial, com análises quantitativas que suportam as conclusões ao fim (KOCHE, 2011). Para a confecção do concreto, foi utilizado o cimento Portland CPII- F32 em função da versatilidade para aplicações e da disponibilidade no mercado local. Para a caracterização do material, utilizou-se a NBR 16605 (ABNT, 2017) por meio da qual encontrou-se massa específica de $3,10 \mathrm{~g} / \mathrm{cm}^{3}$. Como agregado graúdo, empregou-se seixo de origem arenítica adquirido na região metropolitana de Belém, cuja caracterização foi realizada pela execução dos ensaios de: determinação da massa unitária e índice de vazios (ABNT NM 45, 2001); massa específica e absorção de água (ABNT NM 53, 2009); e composição 
granulométrica (ABNT NM 248, 2003), para os quais foram encontrados os valores de 1,499 g/ $/ \mathrm{cm}^{3}, 42,11 \%, 2,623 \mathrm{~g} / \mathrm{cm}^{3} \mathrm{e}$ $1,02 \%$, respectivamente. A dimensão máxima característica e o módulo de finura encontrados para o material foram $19 \mathrm{~mm} \mathrm{e}$ 4,04. A composição granulométrica é apresentada na Tabela 1, onde é possível verificar que as peneiras com aberturas de $9.50 \mathrm{~mm}, 4.80 \mathrm{~mm}$ e $2.40 \mathrm{~mm}$ apresentaram os maiores percentuais de material retido. Não foi utilizado agregado miúdo neste estudo. A água utilizada é oriunda da rede de abastecimento da UFPA, a qual atende aos padrões de potabilidade exigidos pelo comitê brasileiro de cimento e agregados (ABNT/CB 018).

Tabela 1 - Granulometria dos agregados.

\begin{tabular}{ccc}
\hline Peneira & $(\%)$ Retido & $(\%)$ Retido Acumulado \\
\hline 25,00 & 0 & 0 \\
19,00 & 1 & 1 \\
9,50 & 36 & 37 \\
4,75 & 32 & 69 \\
2,36 & 17 & 86 \\
1,18 & 9 & 95 \\
0,60 & 2 & 97 \\
0,30 & 1 & 98 \\
0,15 & 2 & 100 \\
0,075 & 1 & 100 \\
Fundo & 0 & 100 \\
\hline
\end{tabular}

Fonte: Autores (2021).

Para a determinação da proporção de aglomerante, foi aplicado o método proposto por Castro et. al (2009), que parte de parâmetros físicos dos materiais para determinar o fator de compactação (FC), dado pela razão entre a densidade teórica e a real do CPER, cuja necessidade se dá pela ocorrência simultânea de colmatação e criação de vazios adicionais durante os processos de mistura e compactação. Este fator possibilita a determinação do volume de vazios total e de vazios interconectados no estado endurecido, sendo o último, utilizado na obtenção do consumo de cimento (MC). Além do fator de compactação $(\mathrm{FC})$ e do volume de vazios conectados $\left(\mathrm{V}_{\text {con }}\right)$, são relacionados nesta determinação os valores da relação águacimento

$(\mathrm{a} / \mathrm{c})$, do volume de vazios do agregado $\left(\mathrm{V}_{\mathrm{a}}\right)$, da massa específica do cimento $\left(\gamma_{\mathrm{c}}\right)$, da densidade $\left(\mathrm{D}_{\mathrm{ad}}\right)$ e da massa específica do aditivo $\left(\gamma_{\text {ad }}\right)$, quando houver, conforme expresso na equação 1 .

$$
M C(K g)=\frac{(10 V a-100) F C+965,48-11,14 V c o n}{\left(\frac{1}{\gamma c}+\left(\frac{a}{c}\right)+\frac{D a d}{1000 \gamma \mathrm{\gamma d}}\right) F C}
$$

Uma vez definido o consumo de aglomerante, foi possível determinar o consumo de agregado para a mistura relacionando-se a produção de $1 \mathrm{~m}^{3}$ de CPER com sua massa unitária. O material foi utilizado empregando-se a combinação das frações granulométricas retidas nas peneiras $4.80 \mathrm{~mm}$ e $2.40 \mathrm{~mm}$. A escolha de tais frações se deu em função da grande quantidade material disponível das granulometrias e da obtenção de maiores resistências mecânicas utilizando-se grãos menores (Cósic et al.,2015).

As proporções $77 \%$ para grãos de $4.80 \mathrm{~mm}$ e $23 \%$ para $2.40 \mathrm{~mm}$ foram obtidas pelo Método do Empacotamento Compressivo (MEC), proposto por De Larrard (1999). O volume de vazios estabelecido para o CPER no estado endurecido foi 
de $16 \%$, buscando atingir valores aproximados de $2.50 \mathrm{MPa}$ para resistência à tração na flexão e $3 \mathrm{~mm} / \mathrm{s}$ para a taxa de infiltração, conforme indicado por Castro et. al (2009).

A relação água-cimento (a/c) foi determinada a partir do estudo realizado por Ribeiro e Silva (2018), no qual foi investigada a influência da variação da relação água-cimento em concretos permeáveis com seixo rolado, onde o valor de 0,37 apresentou os melhores resultados mecânicos, atendendo aos requisitos de permeabilidade. $\mathrm{O}$ traço obtido é apresentado na Tabela 2.

Tabela 2 - Dosagem dos materiais.

\begin{tabular}{|c|c|c|c|c|}
\hline $\begin{array}{l}\text { Mistura } \\
\text { (c:p) }\end{array}$ & $\begin{array}{c}\text { Consumo de } \\
\text { Cimento }\left(\mathrm{kg} / \mathrm{m}^{3}\right)\end{array}$ & $\begin{array}{c}\text { Consumo de agregado } \\
\qquad\left(\mathrm{kg} / \mathrm{m}^{3}\right)\end{array}$ & Relação a/c & $\begin{array}{c}\text { Teor de pasta } \\
(\%)\end{array}$ \\
\hline $1: 4,25$ & 356,76 & 1499,00 & 0,37 & 24,00 \\
\hline
\end{tabular}

Fonte: Autores (2021).

A fim de avaliar o efeito da ordem de mistura no comportamento do concreto permeável foram elaboradas três sequências de misturas que são: Sequência de Referência (SR), Sequência A (SA) e Sequência B (SB), conforme apresentado na Figura 1. Como misturador foi utilizada uma betoneira de eixo inclinado, cuja capacidade é 150 litros. Foram adotados como valores fixos o traço, a relação água-cimento $(\mathrm{a} / \mathrm{c})$ e o tempo de mistura. O tempo foi definido a partir de ajustes realizados no laboratório para o tempo da sequência de mistura de referência (SR), totalizando 2 minutos.

Figura 1 - Comparativo entre as sequências de mistura.

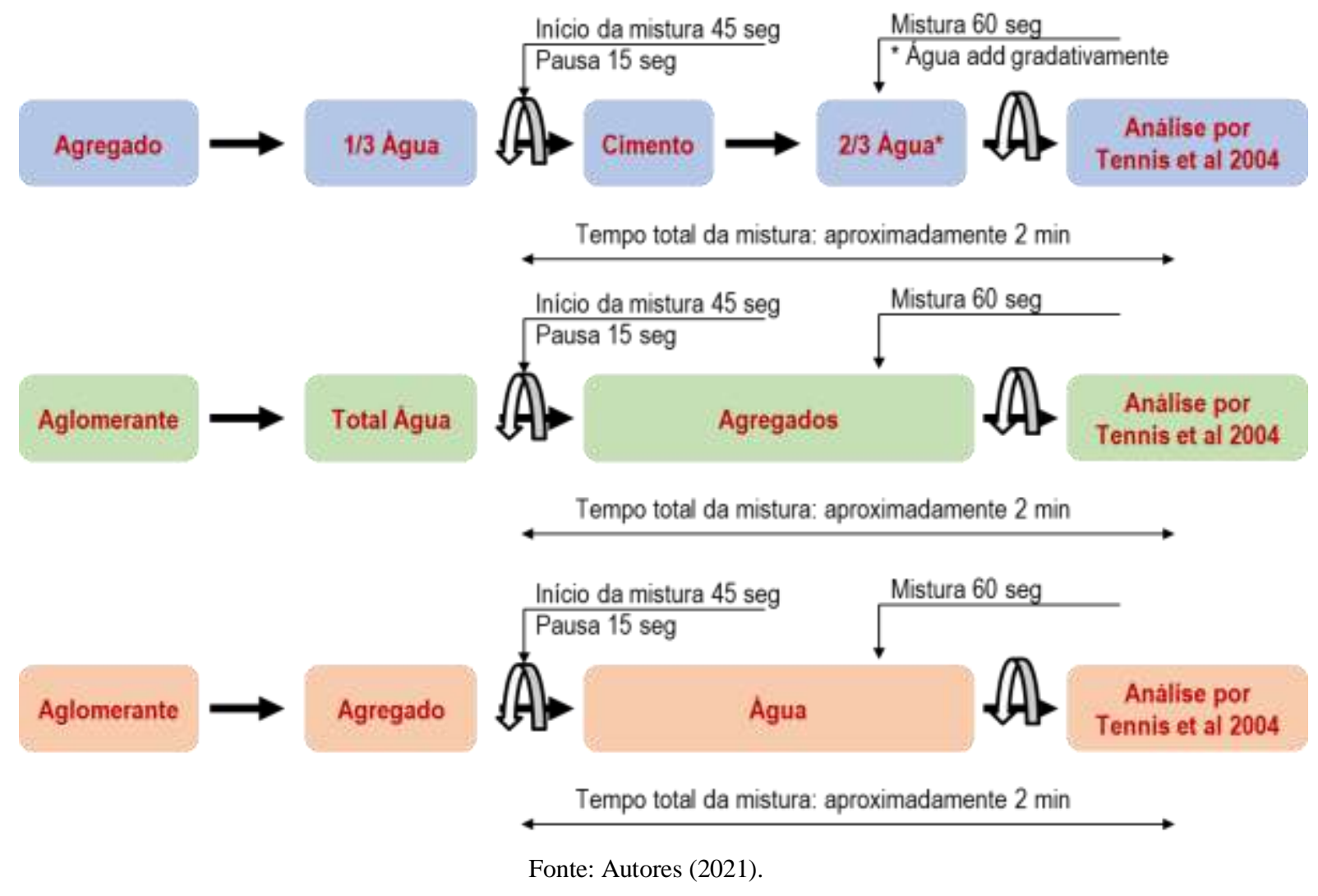


Tomou-se como sequência de referência (SR) uma variação da utilizada na produção de concreto de alto desempenho, utilizada por Neto et. al (2011), que consiste em adicionar o agregado à betoneira juntamente com 1/3 da água. O material é misturado por 45 segundos seguido por uma pausa, em seguida, é adicionado o aglomerante e 2/3 da água, então o material é misturado por 60 segundos.

A Sequência A (SA) é a utilizada por Chindaprasirt et. al (2008) na produção de concreto permeável, que consiste na adição do cimento e da água à betoneira, os quais são misturados por 45 segundos, seguidos por uma pausa para retirada do material das paredes do misturador. Em seguida, são adicionados à pasta os agregados, os quais são misturados por 60 segundos.

A Sequência B (SB) é utilizada na produção de concretos de alta resistência, empregada por Laskar et. al (2011). A sequência consiste na adição dos agregados e do aglomerante à betoneira e estes são misturados por 45 segundos, seguidos por uma pausa. Então, adiciona-se a água de amassamento e o material é misturado por 60 segundos.

Para cada sequência foram moldadas três placas com dimensões de $40 \mathrm{~cm}$ x $40 \mathrm{~cm} \times 10 \mathrm{~cm}$, compactadas utilizando-se um rolo metálico de $55 \mathrm{~kg}$. Durante a compactação, o rolo foi passado sete vezes (ida e volta) sobre as placas de CPER, a fim de empregar a energia de compactação de 0,08MPa, conforme indicado por Castro et. al (2009). O desmolde das placas foi realizado 48 horas após a concretagem, posteriormente, estas passaram pelo processo de cura úmida por imersão durante 28 dias, após os quais foram realizados os ensaios para a avaliação dos comportamentos hidráulico e mecânico.

Para a avaliação do efeito da sequência de mistura no estado fresco do CPER observou-se o cobrimento dos grãos e as condições da pasta quanto à ocorrência de exsudação. Durante a produção das misturas, o material também foi analisado segundo o método proposto por Tennis (2004), que consiste na formação de uma esfera utilizando as mãos a fim de verificar a coesão do material.

Seguindo o apresentado na NBR 16416 (ABNT, 2015) que dita parâmetros sobre pavimentos permeáveis de concreto, também foram avaliados os comportamentos mecânico e hidráulico do material. Foi realizado o tratamento estatístico dos resultados visando correlacionar as variáveis e verificar se as diferenças obtidas entre as misturas foram significativas.

Para a avaliação do comportamento mecânico, foi realizado o ensaio de resistência à tração na flexão, a partir da metodologia de ensaio apresentada na NBR 12142 (ABNT, 2010), uma vez que a finalidade das placas é aplicação para tráfego de veículos leves. Foram ensaiados doze corpos de prova prismáticos com dimensões $10 \mathrm{~cm}$ x $10 \mathrm{~cm}$ x $40 \mathrm{~cm}$ para cada sequência de mistura estudada.

$\mathrm{Na}$ análise hidráulica, foi realizado o ensaio para a determinação do coeficiente de permeabilidade pelo método do anel de infiltração, conforme indicado pela NBR 16416 (ABNT, 2015). O ensaio consiste na fixação de um anel de $30 \mathrm{~cm}$ de diâmetro sobre a placa, o qual deve ter a área de contato vedada, sobre este anel é despejada água, cuja massa depende do tempo de pré-molhagem, durante o lançamento é medido o tempo necessário para que toda a massa de água infiltre. A determinação do coeficiente de permeabilidade $(\mathrm{k}) \mathrm{em} \mathrm{mm} / \mathrm{h}$ é feita relacionando o diâmetro do cilindro (d) em milímetros, o tempo necessário para a percolação da água (t) em segundos, a massa de água (m) em quilogramas e um fator de conversão de unidades (C), conforme expresso na equação 2.

$$
k=\frac{C \cdot m}{\left(d^{2} \cdot t\right)}
$$

As porosidades (total e efetiva) dos concretos também foram calculadas para a análise do desempenho hidráulico, utilizando-se cubos de dimensões $10 \mathrm{~cm} \times 10 \mathrm{~cm} \times 10 \mathrm{~cm}$ extraídos das placas. Para determinar a porosidade total (vt), 
empregou-se a equação 3 (XU et al.,2018), na qual são relacionadas as densidades reais ( $\rho$ s) e teórica ( $\rho t)$, ambas expressas em $\mathrm{g} / \mathrm{cm}^{3}$. Para determinar a porosidade efetiva (ve) são relacionadas as massas seca (m1) e saturada (m2) da amostra em gramas, determinadas a partir da secagem em estufa e submersão por 24 horas, o volume (v) da amostra em centímetro cúbico e a massa específica da água ( $\rho w)$ em gramas por centímetro cúbico, conforme expresso na equação 4.

$$
\begin{gathered}
v t=\left(1-\frac{p s}{p t}\right) 100 \\
v e=\left(1-\frac{m 1-m 2}{v \cdot p w}\right) 100
\end{gathered}
$$

A densidade teórica do concreto ( $\rho t$ ) é obtida a partir da equação 5 (Zheng, 2004), na qual são relacionados parâmetros físicos do aglomerante, do agregado e da água. Eq. 4.

$$
p t=\left(\frac{100+P C+0,25 P C}{\frac{100}{p}+\left(\frac{P C}{p c}\right)+(0,25 p c \cdot 0,75)}\right) p w \quad \text { Eq. } 5
$$

Onde $\rho, \rho \mathrm{w}$ e $\rho \mathrm{c}$ são as massas específicas do agregado, da água e do cimento, respectivamente, e PC é a razão cimento - agregado, em massa.

Analisou-se também a distribuição superficial e a área dos poros por meio do tratamento binário de imagens das faces dos cubos, realizado com o software Image $J$.

Utilizando o método proposto por Matyka e Koza (2012), foi determinada a tortuosidade do CPER para cada sequência de mistura estudada, expresso pela equação 6. Essa análise possibilita compreender a relação entre os processos de transporte no meio e a distribuição dos poros, onde T é a tortuosidade do material, e $\varphi$ é a porosidade.

$$
T=1-\ln (\varphi) \quad \text { Eq. } 6
$$

\section{Resultados e Discussão}

$\mathrm{Na}$ avaliação do efeito da ordem de misturas nas características do concreto permeável no estado fresco, observa-se que as amostras não sofreram exsudação, atribui-se este resultado a fixação do traço, do tempo de mistura e da compactação. $\mathrm{O}$ cobrimento dos agregados foi satisfatório para todas as misturas, o que indica que a mudança na sequência de mistura não influenciou nestes parâmetros. Quanto à coesão, todas as misturas permitiram a formação da esfera proposta por Tennis (2004), conforme apresentado na Tabela 3. O comportamento semelhante das misturas na avaliação pelo método de Tennis (2004) mostra que este não é um bom parâmetro para prever diferenças no comportamento do material no estado endurecido. 
Tabela 3 - Análise das misturas no estado fresco.

\begin{tabular}{l|c|c|c}
\hline $\begin{array}{l}\text { Sequência de } \\
\text { mistura }\end{array}$ & Aspecto Visual & Coesão & $\begin{array}{c}\text { Cobrimento dos } \\
\text { grãos }\end{array}$ \\
\hline SR & & Sim & Satisfatória \\
\hline SA & & Sim & Satisfatória \\
\hline SB & & Sim & \\
\hline
\end{tabular}

Fonte: Autores (2021).

No estado endurecido, os concretos apresentaram cobertura satisfatória dos agregados e não foi possível identificar efeito parede nas faces das placas, entretanto, é possível verificar sua ocorrência nas regiões onde estas foram cortadas para a extração dos prismas e cubos.

Quanto ao comportamento mecânico, a NBR 16416 (ABNT, 2015) estabelece que a resistência mínima à tração na flexão seja $2 \mathrm{MPa}$. Todas as sequências apresentadas neste estudo atenderam, na média, ao requisito de resistência mínima, conforme apresentado na Figura 2. A partir da análise estatística, verificou-se que não existem diferenças significativas entre os resultados, indicando que a mudança na sequência de mistura não provoca grandes variações no comportamento mecânico do material. 
Figura 2 - Resistência à tração na flexão média obtida para cada sequência.

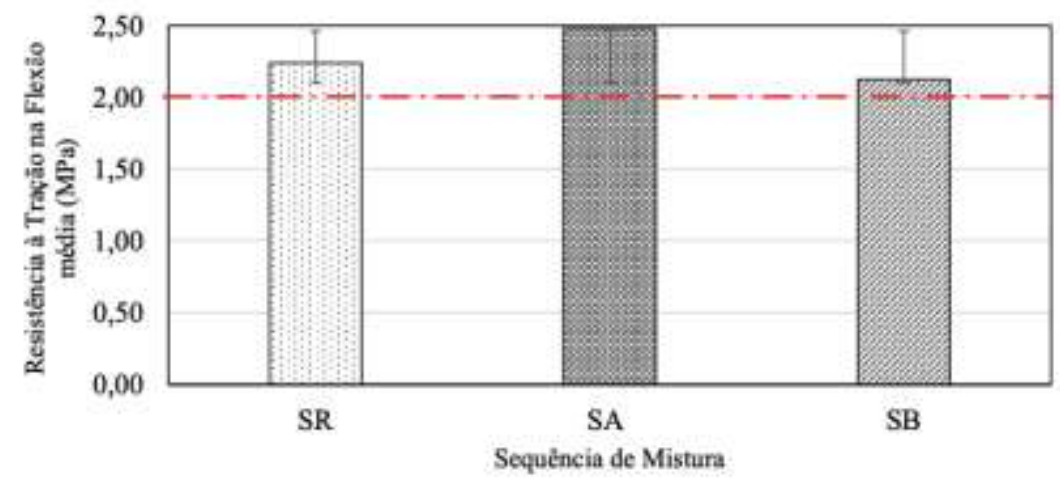

Fonte: Autores (2021).

A partir dos resultados obtidos para o coeficiente de permeabilidade (K), expressos na Figura 3, é possível verificar que todas as sequências atenderam ao requisito normativo para a permeabilidade, $1,0 \mathrm{~mm} / \mathrm{s}$. A partir da análise estatística, observou-se que as diferenças entre permeabilidades nos concretos não foram significativas $(\mathrm{P}>0,05)$, indicando que a sequência de mistura não impacta de forma considerável na permeabilidade do CPER.

Figura 3 - Coeficiente de permeabilidade médio obtido para cada sequência de mistura.

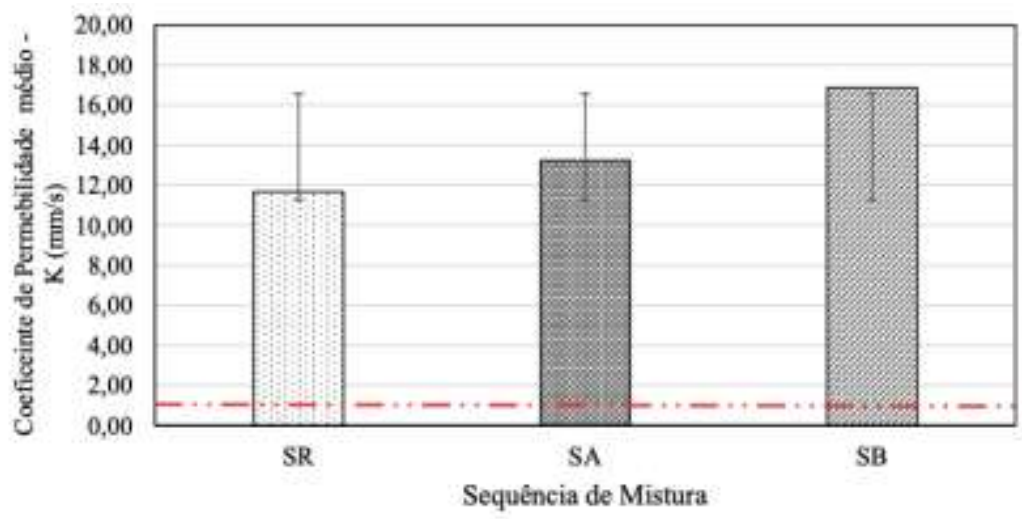

Fonte: Autores (2021).

Dada a finalidade do material, realizou-se a avaliação da quantidade, volume e distribuição dos vazios na superfície da estrutura. Na Figura 4, são apresentadas as faces dos concretos submetidas ao corte, onde as áreas escuras são os poros e as áreas destacadas em vermelho, a visualização do efeito parede. A partir do tratamento realizado pelo software image J, é possível notar o aumento da quantidade de poros para as sequências SA e SB em relação à de referência. As áreas obtidas para os poros das sequências SR, SA e SB foram, respectivamente, 101,25 mm², 203,26mm² e 254,33mm², resultado coerente com as diferenças obtidas nos valores de permeabilidade. 
Figura 4 - Distribuição de vazios superficiais. a) SR; b) SA; c) SB.

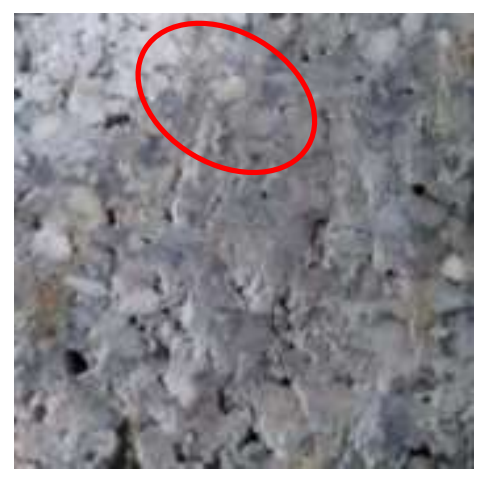

a)

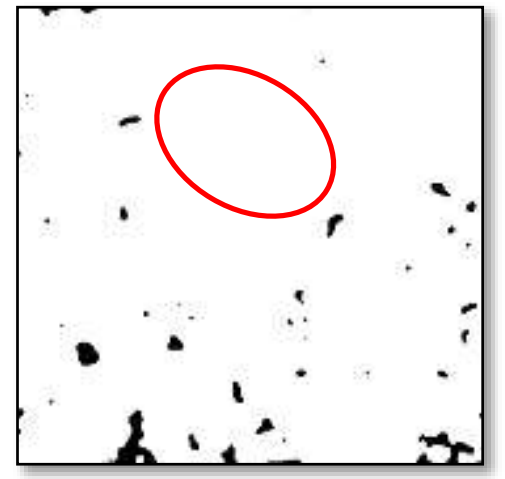

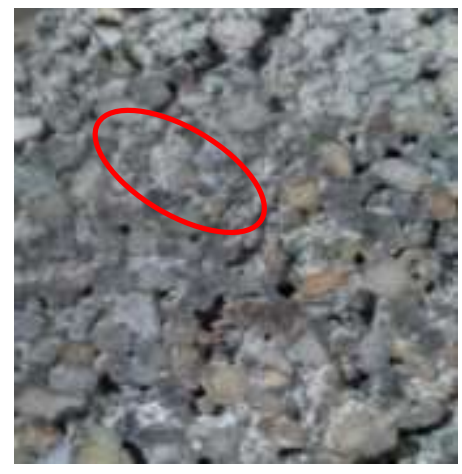

b)

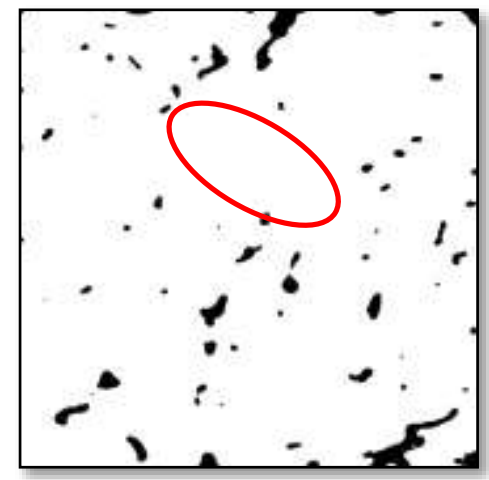

Fonte: Autores (2021).

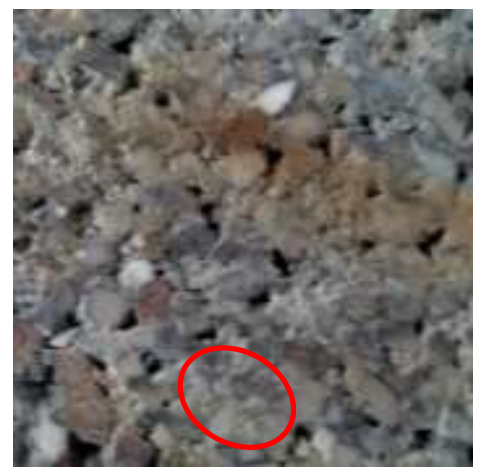

c)

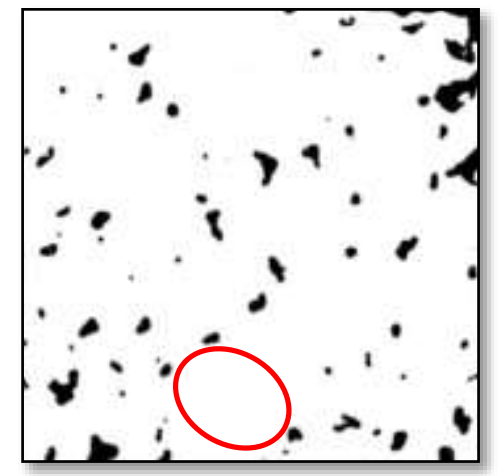

\section{(n)}

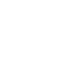

Uma vez visualizados e quantificados os poros, analisou-se o comportamento da porosidade efetiva em relação à total dos concretos, apresentado na Figura 5. É possível observar que a alteração da sequência de mistura durante a produção provocou o aumento das porosidades total e efetiva na sequência $\mathrm{B}$ em relação às demais, o que indica que esta forneceu ao CPER maior quantidade de vazios conectados, resultado coerente com o obtido em termos de permeabilidade. A correlação entre tais parâmetros apresentou valor de $\mathrm{R}^{2}=0,783$, devido ao baixo consumo de agregado, quando comparado à valores encontrados na literatura internacional, o que possibilita a formação de efeito parede em regiões de concentração de pasta.

Figura 5 - Relação entre porosidade total e porosidade efetiva.

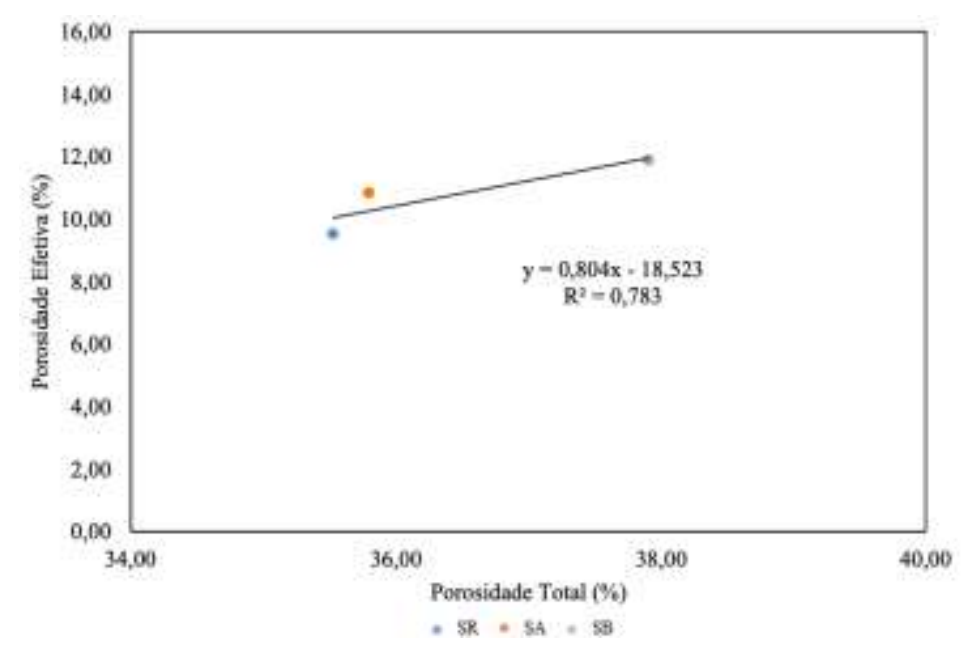

Fonte: Autores (2021). 
Os parâmetros de porosidades totais e efetivas influenciam diretamente na tortuosidade, ou seja, na resistência que o CPER exerce sobre a percolação da água. Na Figura 6 é possível verificar o comportamento da porosidade efetiva em relação aos valores de tortuosidade encontrados para cada sequência estudada. Nota-se que quanto maiores os valores de tortuosidade, maiores os de porosidade efetiva, uma vez que esta está relacionada com o caminho dos poros interconectados.

Figura 6 - Relação entre porosidade efetiva e Tortuosidade.

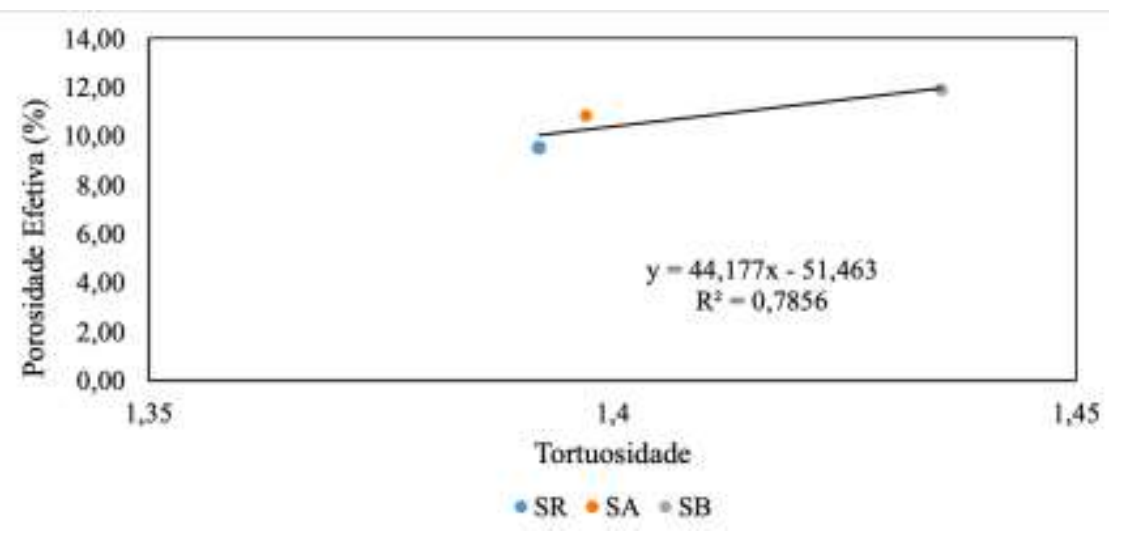

Fonte: Autores (2021).

Também foi verificado o comportamento do coeficiente de permeabilidade em relação à porosidade efetiva. Na Figura 7 é possível verificar que existe coerência entre esta correlação e os demais resultados. Observa-se que K cresce exponencialmente em função do aumento da porosidade efetiva, correlação que apresenta $\mathrm{R}^{2}$ com valor superior ao obtido por Xu et. al (2018).

Figura 7 - Correlação entre o coeficiente de permeabilidade médio e a porosidade efetiva.

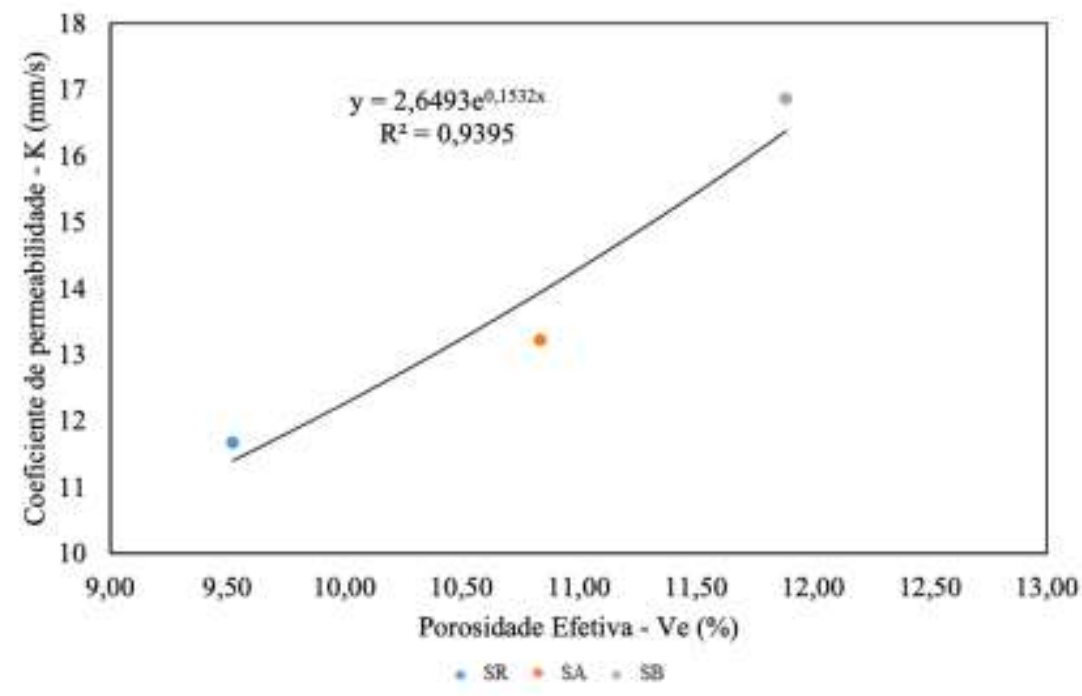

Fonte: Autores (2021).

Por fim, a última correlação analisada foi entre a porosidade do material e sua resistência à tração na flexão. A Figura 8 apresenta o comportamento CPER frente ao aumento do volume de poros. Nota-se que as misturas SR e SA, mais compactas, apresentaram resultados de resistência superiores à mistura SB, uma vez que este parâmetro é o que maior impacta na sobre 
suas propriedades mecânicas, conforme apresentado em Zhong et al. (2016). O resumo dos resultados pode ser observado na Tabela 4.

Figura 8 - Comportamento da Resistência à tração na flexão média em função da porosidade total.

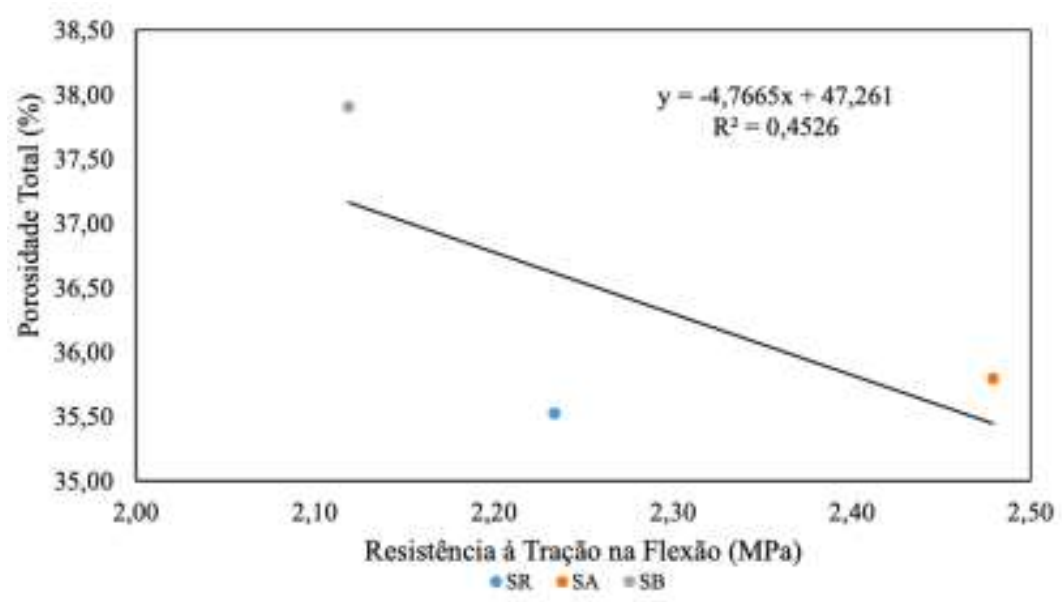

Fonte: Autores (2021).

Tabela 4 - Resumo dos resultados obtidos para cada sequência estudada.

\begin{tabular}{ccc}
\hline Ensaio & Amostra & Resultados \\
\hline Resistência à tração na flexão & SR & 2,23 \\
média (MPa) & SA & 2,48 \\
Coeficiente de & SB & 2,11 \\
Permeabilidade Médio $-\mathrm{K}$ & SR & 11,67 \\
(mm/s) & SA & 13,22 \\
& SB & 16,86 \\
Porosidade Total $(\%)$ & SR & 35,52 \\
& SA & 35,79 \\
Porosidade Efetiva $(\%)$ & SB & 37,90 \\
& SR & 9,52 \\
Tortuosidade & SA & 10,84 \\
& SB & 11,88 \\
& SR & 1,392 \\
& SA & 1,397 \\
& SB & 1,435 \\
\hline
\end{tabular}

Fonte: Autores (2021).

\section{Conclusão}

Com relação ao efeito da ordem de mistura no comportamento hidráulico e mecânico do material, conclui-se que pelas três ordens apresentadas é possível formular concretos que atendam as características da NBR 16416 (ABNT, 2015), para aplicação em pavimentos permeáveis para o tráfego leve.

Os concretos não sofreram exsudação, e na face externa não se observou efeito parede. Porém, ao seccionar as placas 
observou-se em algumas misturas regiões internas com concentração de pasta, que afetou na relação entre o volume e distribuição de poros.

Os resultados obtidos a partir da sequência B apresentaram os melhores valores, indicando que esta forneceu a melhor geometria, aumentando assim a capacidade drenante do material. Para trabalhos futuros, sugere-se variar as ordens de dosagem com outros agregados graúdos diferentes do seixo, ou ainda uma análise com diferentes métodos de dosagem na literatura para concretos permeáveis.

\section{Referências}

ABNT (2015) - NBR 16416: Pavimentos permeáveis de concreto - Requisitos e procedimentos. Associação Brasileira de Normas Técnicas.

ABNT (2003) - NBR NM 248: Agregados - Determinação da composição granulométrica. Associação Brasileira de Normas Técnicas.

ABNT (2006) - NBR NM 45: Agregados - Determinação da massa unitária e do volume de vazios. Associação Brasileira de Normas Técnicas.

ABNT (2009) - NBR NM 53: Agregados graúdo - Determinação da massa específica, massa específica aparente e absorção de águas. Associação Brasileira de Normas Técnicas.

ABNT (2010) - NBR 12142: Concreto - Determinação da resistência à tração de corpos de prova prismáticos. Associação Brasileira de Normas Técnicas.

Agar-Ozbek, A. S., Weerheijm, J., Schlangen, E. \& Van Breugel, K. (2013). Investigating porous concrete with improved strength: Testing at different scales. Revista. Construction and Building Materials. 41, 480-490.

Batezini, R. (2013). Estudo preliminar de concretos permeáveis como revestimento de pavimentos para áreas de veículos leves. Dissertação de mestrado apresentada à Escola Politécnica da Universidade de São Paulo.

Bhutta, M.A.R., Tsuruta, K. \& Mirza, J. (2012). Evaluation of high-performance porous concrete properties. Construction and Building Materials. 31 , 67-73.

Bradley, P. \& Andrew, N. (2011). Comparison of test specimen preparation techniques for pervious concrete pavements. Construction and Building Materials. $25,3480-3485$.

Castro, J.,Solminihac, H., Videla, C. \& Fernandéz, B, (2009). Estudio de dosificaciones en laboratorio para pavimentos porosos de hormigón. Revista Ingenieria de Construccion. 24(3), 271-284.

Chandrappa, A. K. \& Biligiri, K. P. (2018). Pervious concrete as a sustainable pavement material - Research findings and future prospects: A state-of-theart review. Construction and Building Materials. 111, 262-274.

Chen, Y., Wang, K. \& Liang, D. (2012). Mechanical Properties of Pervious Cement Concrete, J. Cent. South Univ. 19, $3329-3334$.

Chindapraist, P., Hatanaka, S., Chareerat, T., Mishima, N. \& Yuasa, Y. (2008). Cement paste characteristics and porous concrete properties. Construction and Building Materials. Vol. 22, p. 894-901.

De Larrard, F. (1999). Concrete Mixture Proportioning - A Scientific Approach. Modern Concrete Technology Series, 9 , E\&FN SPON, 421 p.

Filho, M. (2017) Determinação da tortuosidade de arenitos e carbonatos utilizando florestas de caminhos ótimos. Tese de Doutorado apresentada à Uviversidade do Estadual de Campinas.

Koche, J. C. (2011). Fundamentos de metodologia científica. Petrópolis.

Laskar, I. (2011) Mix design of high-performance concrete. Materials Research. 14 (4), 429-433.

Lian, C., Zhuge, Y. \& Beecham, S. (2011). The relationship between porosity and strength for porous concrete. Construction and Building Materials. 25, 4294-4298.

Masoud, K., Dane, A., John T. \& Eta, A. (2012). Permeability measurement and scan imaging to assess clogging of pervious concrete pavements in park lots. Construction and Building Materials. 95, 114-123.

Matyka, M. \& Koza, Z. (2012). How to Calculate Tortuosity Easily? AIP Conference Proceedings 1453, 17.

Mehta, P. K. \& Monteiro, P. (2008). Concreto: microestrutura, propriedades e materiais. (3a ed.) Ibracon.

Neto, B., Oliveira, C. \& Ramos, D. (2011). Efeitos do tipo, tamanho e teor de agregado graúdo no módulo de deformação do concreto de alta resistência. Revista matéria, 16 (2), 680-702.

Nicholas A., Hamid A. \& Fatih A. (2016). Flexural strength and fracture size effects of pervious concrete. Construction and Building Materials. 113, 536-543.

Salomão, P. E. A., Porto, T. B., Cabrai, S. C., da Silva, W. L., \& de Oliveira, A. N. S. (2018). Elaboration of tables for concrete dosage based on the aggregates used in Northeast Mineiro. Research, Society and Development, 7 (4), 1274305 
Research, Society and Development, v. 10, n. 4, e31110414205, 2021

(CC BY 4.0) | ISSN 2525-3409 | DOI: http://dx.doi.org/10.33448/rsd-v10i4.14205

Tennis, P., Leming, M. \& Akers, D. (2004). Pervious concrete pavements. Portland Cement Association, Skokie, Illinois, and National Ready Mixed Concrete Association, Silver Spring, Maryland, USA.

Torres, A., Hu, J. \& Ramos, A. (2015). The effect of the cementitious paste thickness on the performance of pervious concrete. Construction and Building Materials. 95, 850-859.

Tucci, C. (2002). Gerenciamento da drenagem urbana. Revista Brasileira de recursos hídricos, 7, 5-27.

Tucci, C. (2009). Hidrologia: ciência e aplicação. Revista Brasileira de recursos hídricos, 07, 5-27.

Xu, G., Shen, W., Huo, X., Yang, Z.,Wang, J., Zhang, W. \& Ji, X. (2018). Investigation on the properties of porous concrete as road base material. Construction and Building Materials. 158, 141-148.

Zheng, M. (2004). Physical And Mechanical Performance Of Porous Concrete For Drainage Base. Tese De Pós-Doutorado. China. 